\title{
Effects of Nogo-A receptor antagonist on the regulation of the Wnt signaling pathway and neural cell proliferation in newborn rats with hypoxic ischemic encephalopathy
}

\author{
YONGXIANG WANG ${ }^{*}$, JIAXIANG GU* ${ }^{*}$ XINMIN FENG, HUA WANG, YUPING TAO and JINGCHENG WANG \\ Department of Orthopedic Surgery, Clinical Medical College of Yangzhou, University of Yangzhou, \\ Yangzhou, Jiangsu 225001, P.R. China
}

Received February 16, 2013; Accepted July 3, 2013

DOI: $10.3892 / \mathrm{mmr} .2013 .1579$

\begin{abstract}
Hypoxic ischemic encephalopathy is a serious condition due to inadequate oxygen supply to the brain. Regeneration of neural cells is a critical process for repairing the damaged brain. Nogo has been identified as an inhibitor of neurite outgrowth that is specific to the brain. In the present study, the Nogo-A receptor (NgR) antagonist NEP1-40 was used to study the effects of inhibition of $\mathrm{NgR}$ on the regeneration of neural cells and the related Wnt signaling pathway in newborn rats. The investigation focused on the transcription factors regulated in the Wnt signaling pathway during the repair process, together with the proliferation of neural cells. The results indicated that c-Jun and c-Myc were the main transcription factors involved in the Wnt signaling pathway, while neural cell proliferation in the subventricular zone was increased during this process.
\end{abstract}

\section{Introduction}

Hypoxic ischemic encephalopathy (HIE) is a serious condition due to the inadequate oxygen supply to the brain, and is associated with oxygen deprivation in the neonate. For HIE neonates, regeneration of neural cells is a critical process for repairing the damaged brain (1). Several inhibitors are capable of reducing the ability of central nervous system (CNS) repair, among which Nogo A is important (2). Nogo has been identified as an inhibitor of neurite outgrowth that is specific to the CNS. It belongs to the family of reticulon-encoding genes, associated with the endoplasmic reticulum (3). Nogo is a potent neurite outgrowth inhibitor that may also block the regeneration of

Correspondence to: Professor Jingcheng Wang, Department of Orthopedic Surgery, Clinical Medical College of Yangzhou, University of Yangzhou, Yangzhou, 98 West Nantong Road, Jiangsu 225001, P.R. China

E-mail:0001wjc@sina.com

*Contributed equally

Key words: hypoxic ischemic encephalopathy, Wnt signaling pathway, Nogo-A, NEP1-40, neural cell proliferation the CNS in higher vertebrates (4). Nogo-66 receptor ( $\mathrm{NgR})$, together with oligodendrocyte myelin glycoprotein and myelinassociated glycoprotein, mediate axonal growth inhibition and may play a role in regulating axonal regeneration and plasticity in the adult CNS (5). However, NgR may inhibit the regeneration of neurons and related nervous cells in HIE neonates and thus hinder the repair of injured CNS. NgR mediates axonal growth inhibition and may play a role in regulating axonal regeneration and plasticity in the adult CNS (6).

The Wnt signaling pathway controls cell-cell communication in the embryo and in adults, including cell proliferation and differentiation during development and healing (7). A previous study indicated that the Wnt signaling pathway is involved in regeneration of neural cells mediated by Nogo; however, the mechanism involved remains unknown (8). Inhibition of $\mathrm{NgR}$ is considered as one potentially useful method for treatment of HIE neonates. To study the effects of inhibition of $\mathrm{NgR}$ on the regeneration of injured $\mathrm{CNS}$ and the related transcription factors (TFs) involved, Nogo-A receptor antagonist NEP1-40 was used in the present study. The investigation focused on the TFs in the Wnt signaling pathway that are regulated by inhibition of $\mathrm{NgR}$ during CNS regeneration, together with the proliferation of neural cells.

\section{Materials and methods}

Generation of animal model and drug treatments. Newborn

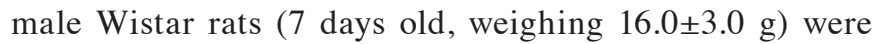
provided by the Animal Research Center, University of Yangzhou, China. The newborn hypoxic ischemic encephalopathy rat animal model was generated as described previously and rats were named hypoxic ischemic brain damage (HIBD) rats (9). The 40 HIBD rats were divided into a HIBD group and a HIBD + NEP1-40 group ( $n=20$ in each group). The HIBD + NEP1-40 group rats were treated with NEP1-40 for 7 days as previously described (9). Rats were sacrificed by inhalation of $\mathrm{CO}_{2}$ for $3 \mathrm{~min}$. This study was approved by the Medical Ethics Committee of the Clinical Medical College of Yangzhou, University of Yangzhou (Yangzhou, China).

Quantitative PCR ( $q P C R)$. Total RNA from rat brains was isolated using TRIzol (Invitrogen Life Technologies, Carlsbad, 


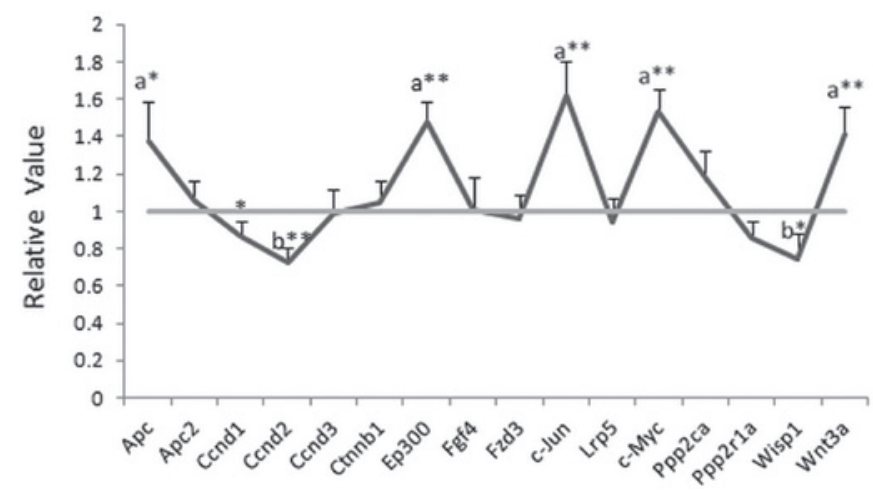

Figure 1. Gene expression in the HIBD and HIBD + NEP1-40 groups. Values in the HIBD group were set as 1 in all groups and the relative value was calculated by comparing the HIBD + NEP1-40 group with the HIBD group. a: $>1.35$-fold; b: $<0.75$-fold. ${ }^{*} \mathrm{P}<0.05 ;{ }^{* *} \mathrm{P}<0.01$ in comparison between HIBD and HIBD + NEP1-40 groups. HIBD, hypoxic ischemic brain damage.

CA, USA) according to the manufacturer's instructions. The Rat WNT Signaling Pathway PCR array (SABiosciences, Qiagen, Inc., Frederick, CA, USA), which contained Apc, Apc2, Ccnd1, Ccnd2, Ccnd3, Ctnnb1, Ep300, Fgf4, Fzd3, c-Jun, Lrp5, c-Myc, Ppp2ca, Ppp2r1a, Wisp1 and Wnt3a was used to detect the expression of genes related to the Wnt signaling pathway. After reverse transcription using a cDNA Synthesis kit (Invitrogen Life Technologies), all the products were used as the templates for the qPCR using the ABI Prism SDS 7000 (Applied Biosystems, Inc., Foster City, CA, USA). qPCR conditions were as follows: i) $50^{\circ} \mathrm{C} 2 \mathrm{~min}, 1$ cycle; ii) $95^{\circ} \mathrm{C} 10 \mathrm{~min}, 1$ cycle; iii) $95^{\circ} \mathrm{C} 15 \mathrm{sec}$, followed by $60^{\circ} \mathrm{C}$ $30 \mathrm{sec}$ and $72^{\circ} \mathrm{C} 30 \mathrm{sec}, 40$ cycles; iv) $72^{\circ} \mathrm{C} 10 \mathrm{~min}, 1$ cycle.

Western blot analysis. Total protein extracted from rat brains $(12 \mu \mathrm{g})$ was boiled at $100^{\circ} \mathrm{C}$ with $4 \mathrm{X}$ loading buffer for $5 \mathrm{~min}$, and then subjected to $10 \%$ SDS-PAGE (Invitrogen Life Technologies). After electrophoresis, the gel was transferred onto a nitrocellulose (NE) membrane at $70 \mathrm{~V}$ for $2 \mathrm{~h}$ at $4^{\circ} \mathrm{C}$. After blocking in 5\% nonfat milk for $1 \mathrm{~h}$, the membrane was incubated with Apc (1:800, rabbit polyclonal IgG; Millipore, Billerica, MA, USA), Ep300 (1:1,000, rabbit polyclonal IgG; Sigma-Aldrich, St. Louis, MO, USA), c-Jun (1:1,000, rabbit polyclonal IgG; Millipore), c- Myc (1:1,000, rabbit polyclonal IgG; Millipore) and Wnt3a (1:1,000, rabbit polyclonal IgG; Millipore) primary antibodies overnight at $4^{\circ} \mathrm{C}$ in $3 \%$ BSA according to the results from qPCR. After washing with $1 \mathrm{X}$ tris phosphate-buffered saline (TPBS; $\mathrm{pH} 7.4$ ), the membrane was incubated with secondary antibody (goat anti-rabbit IgG, Cell Signaling Technology, Inc., Boston, MA, USA) for $1 \mathrm{~h}$ at room temperature, washed again with $1 \mathrm{X}$ TPBS (pH 7.4), and images were captured with film exposure (Kodak, Rochester, NY, USA) for analysis. $\beta$-actin was used as a negative control. The value of each protein was first compared with $\beta$-actin, then the relative value was compared between the HIBD and HIBD + NEP1-40 groups.

Immunofluorescence (IF) for cell proliferation. Brain extracts were detected by IF for the expression of Ki67. Cryostat rat brain coronal sections $(12 \mu \mathrm{m})$ were prepared (Leica, Solms, Germany) and stained with Ki67 antibody (Abcam,

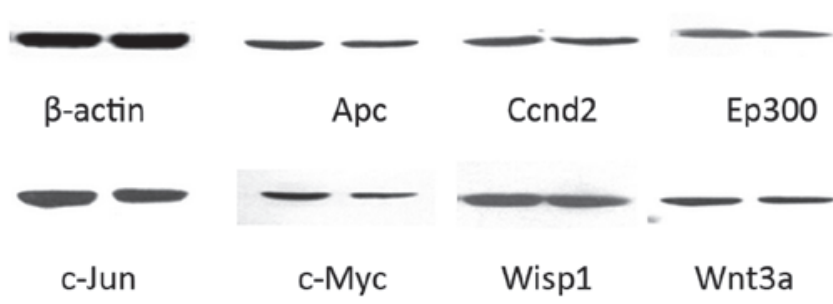

Figure 2. Results of western blot analysis. Left: HIBD + NEP1-40 group; right: HIBD group. HIBD, hypoxic ischemic brain damage.

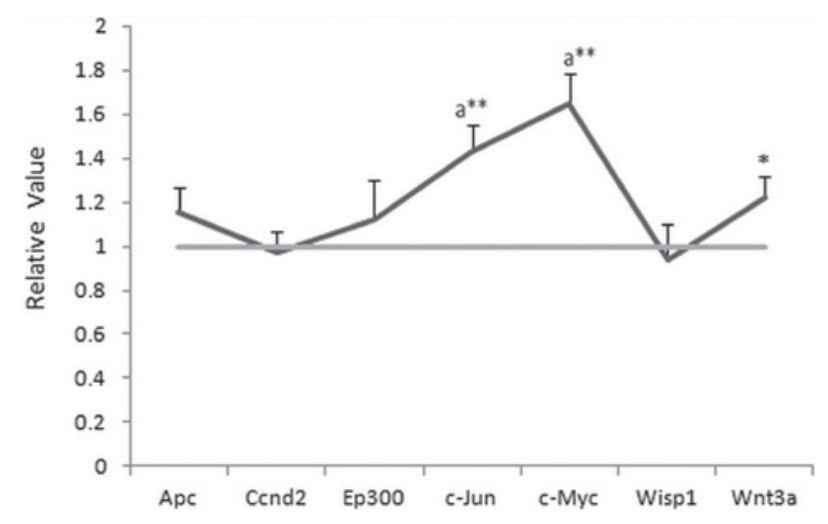

Figure 3. Protein expression in the HIBD and HIBD + NEP1-40 groups Values in the HIBD group were set as 1 in all groups and the relative value was calculated by comparing the HIBD + NEP1-40 group with the HIBD group. (a: $>1.35$-fold; ${ }^{*} \mathrm{P}<0.05$; ${ }^{* *} \mathrm{P}<0.01$ in comparison between HIBD and HIBD + NEP1-40 groups). HIBD, hypoxic ischemic brain damage.

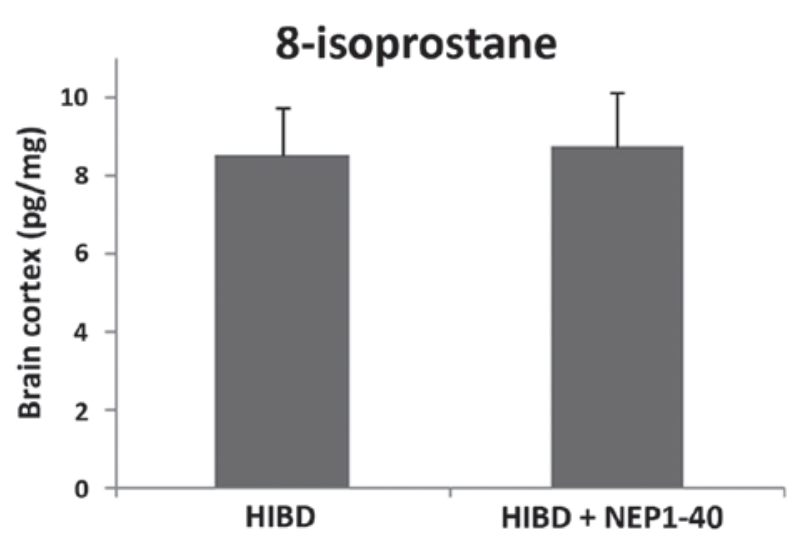

Figure 4. 8-Isoprostane detection in the HIBD and HIBD + NEP1-40 groups. The value in the HIBD group was set as 1 in all groups and the relative value was calculated by comparing the HIBD + NEP1-40 with the HIBD group. No significant changes were detected. HIBD, hypoxic ischemic brain damage.

Cambridge, MA, USA; 1:1,000) to assess the proliferation of neural cells in the subventricular zone (SVZ). Images were captured using a confocal microscope, shown in the dark (A1R MP+ Multiphoton Confocal; Nikon, Tokyo, Japan; x100).

8-Isoprostane detection. The brains were homogenized in TBS buffer with protease inhibitors (1:1,000, Invitrogen Life Technologies) and centrifuged at $12,000 \mathrm{~g} / \mathrm{min}$ for $40 \mathrm{~min}$ at $4^{\circ} \mathrm{C}$. Supernatant was transferred into another tube. The 

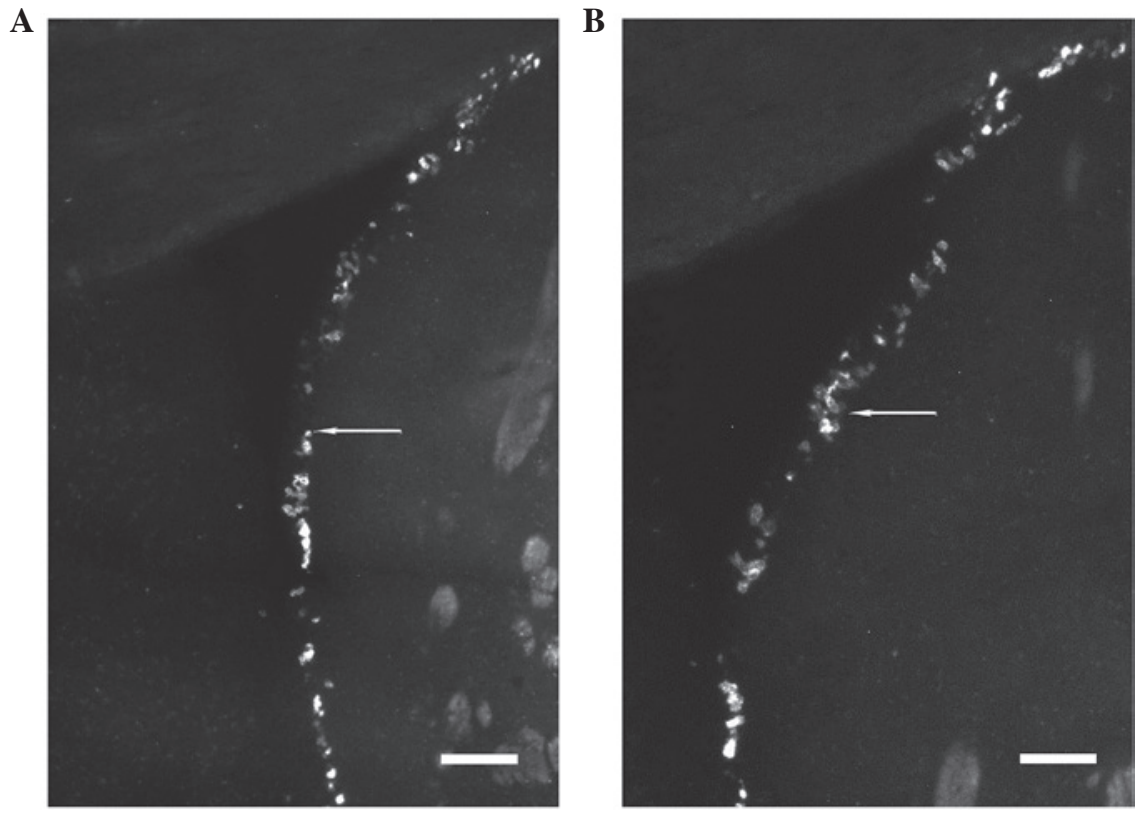

Figure 5. Detection of Ki67 expression in the SVZ in the HIBD and HIBD + NEP1-40 groups (magnification, x200; bar, 200 $\mu \mathrm{m}$ ). (A) HIBD + NEP1-40 group; (B) HIBD group. HIBD, hypoxic ischemic brain damage; SVZ, subventricular zone. Ki67 positive cells are indicated by arrows.

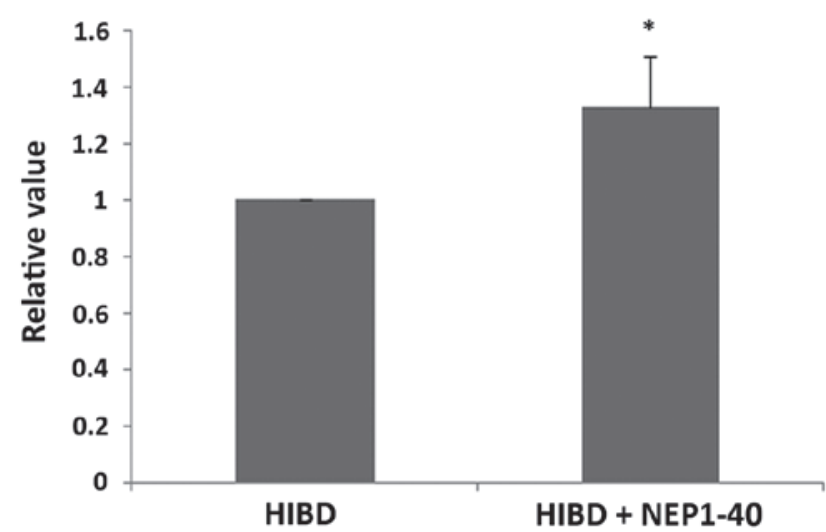

Figure 6. Analysis of Ki67 expression. The value in the HIBD group was set as 1 in all groups and the relative value was calculated by comparing the HIBD + NEP1-40 group with the HIBD group. ${ }^{*} \mathrm{P}<0.05$ in comparison between HIBD and HIBD + NEP1-40 groups. HIBD, hypoxic ischemic brain damage.

levels of 8-isoprostane, a special marker for reactive oxygen species (ROS), were determined using an 8-Isoprostane EIA kit (Cayman Chemical Company, Ann Arbor, MI, USA) according to the manufacturer's instructions.

Statistical analysis. All data were expressed as the means \pm SD. The results were evaluated by Student's t-tests. Statistically significant differences between groups were defined as $\mathrm{P}<0.05$ and $\mathrm{P}<0.01$. Calculations were performed using SPSS 13.0 (SPSS, Inc., Chicago, IL, USA).

\section{Results}

Effects of NEP1-40 on gene expression. The gene expression of Apc, Ep300, c-Jun, c-Myc and Wnt3a was significantly increased ( $>1.35$-fold) while Ccnd 2 and Wisp1 $(<0.75$-fold) were decreased in the HIBD + NEP1-40 group after treatment with NEP1-40 for 7 days. For other genes, no significant changes $(>1.5$ - or $<0.75$-fold) were detected. The value in the HIBD group was set as 1 , while the relative value was calculated by comparing the HIBD + NEP1-40 group with the HIBD group. All data are shown in Fig. 1.

Effects of NEP1-40 on protein expression. As shown in Fig. 2, the expression of c-Jun and c-Myc at the protein level were upregulated ( $>1.5$-fold) after treatment with the Nogo-A receptor antagonist NEP1-40 for 7 days, which correlated with the changes observed for gene expression. However, no marked changes in Apc, EP300, Wnt3a, Ccnd2 and Wisp1 expression $(>1.35$ - or $<0.75$-fold) were detected, in contrast with the gene expression results. The value in the HIBD group was set as 1 , while the relative value was calculated by comparing the HIBD + NEP1-40 group with the HIBD group. All data were analyzed in Fig. 3.

Analysis of 8-isoprostane detection. 8-Isoprostane is an ideal biomarker for detecting oxidative stress in animal tissues and organs. No significant changes in 8-isoprostane were detected between the HIBD + NEP1-40 group and the HIBD group (Fig. 4). This study indicated that the Nogo-A receptor antagonist NEP1-40 did not affect oxidative stress in the CNS during HIE.

Analysis of the regeneration of neural cells. As indicated by arrows showing Ki67 in Fig. 5 (bar, $200 \mu \mathrm{m}$ ), increased regeneration of neural cells was detected in the HIBD + NEP1-40 group (Fig. 5A) compared with the HIBD group (Fig. 5B) in the SVZ, the site of neural cellular proliferation in the adult brain, which may be useful for repair of damage to the CNS. Promotion of neural cellular proliferation is a potential method for HIE treatment. The value in the HIBD group was set as 
1 , while the relative value was calculated by comparing the HIBD + NEP1-40 group with the HIBD group. All data were analyzed in Fig. 6.

\section{Discussion}

Numerous proteins are involved in HIE, including Nogo, which is involved in neuroendocrine secretion or in membrane trafficking in neuroendocrine cells (10). Nogo-A has two known inhibitory domains including amino-Nogo, at the N-terminus, and Nogo-66 (4). Blocking Nogo-A during neuronal damage may help to protect or restore the damaged neurons. $\mathrm{NgR}$ is a high-affinity binding receptor for a region of Nogo, a myelin-associated protein that inhibits axon outgrowth, which requires membrane-spanning co-receptors to transduce growth inhibitory signals (11). $\mathrm{NgR}$ is implicated in neuronal plasticity and regeneration (12). However, the mechanism involved remains unknown, among which the Wnt signaling pathway is valuable to study. Wnt signaling pathways play a variety of roles in embryonic development, cell differentiation and cell polarity generation (13), particularly for shifting ventral genes into dorsal regions of the neural tube (14). In this study, c-Jun and c-Myc were found to be upregulated. c-Jun plays an important role in cellular proliferation and apoptosis of the endometrium throughout the menstrual cycle (15). Cyclical changes of the c-Jun protein levels are significant in the proliferation and apoptosis of glandular epithelial cells (16). c-Myc activates the expression of a number of genes through binding to enhancer box sequences and recruiting histone acetyltransferases (HATs) (17), activated upon various mitogenic signals, including the Wnt signaling pathway (18).

8-Isoprostane is an ideal biomarker of oxidative stress and increased concentrations are detected during this progress, indicating that an imbalance between the systemic manifestation and clearance of reactive oxygen species results in body or organ damage. (19). Our research indicated that no significant change in 8-isoprostane levels was detected between the HIBD + NEP1-40 group and the HIBD group. This result suggested that oxidative stress was not related to or involved in the inhibition of Nogo-A during neuronal damage and neuronal repair. Ki67 is a cellular marker for proliferation. Ki67 is strictly associated with cell proliferation and is present during all active phases of the cell cycle $\left(\mathrm{G}_{1}\right.$, $S, G_{2}$ and mitosis), but is absent from resting cells $\left(G_{0}\right)(20)$. Increased Ki67 expression means that increased regeneration of neural cells was detected in the HIBD + NEP1-40 group in the SVZ, the area of neural cellular proliferation in the adult brain. Promotion of neural cellular proliferation is a potential method for HIE treatment, which requires further study. Given that this antagonist may be a good potential drug target for the treatment of HIE, the importance of this in vivo study is currently under intense investigation.

This study focused on the effects of the Nogo-A receptor antagonist, NEP1-40, on regulation of the Wnt signaling pathway and neural cell proliferation in newborn HIE rats. It was indicated by inhibition of $\mathrm{NgR}$ that c-Jun and c-Myc were the main TFs in the Wnt signaling pathway, while neural cell proliferation in the SVZ was increased during this process.

\section{Acknowledgements}

This study was supported by the National Natural Science Foundation of China (grant no. 81071466) and Research Project Foundation on Social Development of Science and Technology of Yangzhou (YZ2011082).

\section{References}

1. Pietrini D, Piastra M, Luca E, Mancino A, Conti G, Cavaliere F and De Luca D: Neuroprotection and hypothermia in infants and children. Curr Drug Targets 13: 925-935, 2012.

2. Pernet V and Schwab ME: The role of Nogo-A in axonal plasticity, regrowth and repair. Cell Tissue Res 49: 97-104, 2012.

3. Skaper SD: Neuronal growth-promoting and inhibitory cues in neuroprotection and neuroregeneration. Methods Mol Biol 846: 13-22, 2012.

4. Borrie SC, Baeumer BE and Bandtlow CE: The Nogo-66 receptor family in the intact and diseased CNS. Cell Tissue Res 349: 105-117, 2012.

5. McDonald CL, Bandtlow C and Reindl M: Targeting the Nogo receptor complex in diseases of the central nervous system. Curr Med Chem 18: 234-244, 2011.

6. Cao Z, Gao Y, Deng K, Williams G, Doherty P and Walsh FS: Receptors for myelin inhibitors: structures and therapeutic opportunities. Mol Cell Neurosci 43: 1-14, 2010.

7. Budnik V and Salinas PC: Wnt signaling during synaptic development and plasticity. Curr Opin Neurobiol 21: 151-159, 2011.

8. Cerpa W, Toledo EM, Varela-Nallar L and Inestrosa NC: The role of Wnt signaling in neuroprotection. Drug News Perspect 22: 579-591, 2009.

9. Cao Y, Shumsky JS, Sabol MA, Kushner RA, Strittmatter S, Hamers FP, Lee DH, Rabacchi SA and Murray M: Nogo-66 receptor antagonist peptide (NEP1-40) administration promotes functional recovery and axonal growth after lateral funiculus injury in the adult rat. Neurorehabil Neural Repair 22: 262-278, 2008.

10. Llorens F, Gil V and del Río JA: Emerging functions of myelinassociated proteins during development, neuronal plasticity, and neurodegeneration. FASEB J 25: 463-475, 2011.

11. Teng FY and Tang BL: Nogo-A and Nogo-66 receptor in amyotrophic lateral sclerosis. J Cell Mol Med 12: 1199-1204, 2008.

12. Zhang S, Zhang Q, Zhang JH and Qin X: NgR acts as an inhibitor to axonal regeneration in adults. Front Biosci 13: 2030-2040, 2008.

13. Cadigan KM: TCFs and Wnt/ $\beta$-catenin signaling: more than one way to throw the switch. Curr Top Dev Biol 98: 1-34, 2012.

14. Clevers $\mathrm{H}$ and Nusse $\mathrm{R}$ : Wnt $/ \beta$-catenin signaling and disease. Cell 149: 1192-1205, 2012.

15. Otsuki Y: Apoptosis in human endometrium: apoptotic detection methods and signaling. Med Electron Microsc 34: 166-173, 2001.

16. Doucas H, Garcea G, Neal CP, Manson MM and Berry DP: Changes in the Wnt signalling pathway in gastrointestinal cancers and their prognostic significance. Eur J Cancer 41: 365-379, 2005.

17. Honeycutt KA and Roop DR: c-Myc and epidermal stem cell fate determination. J Dermatol 31: 368-375, 2004.

18. Katoh M and Katoh M: WNT signaling pathway and stem cell signaling network. Clin Cancer Res 13: 4042-4045, 2007.

19. Kim HS, Lee K, Kang KA, Lee NH, Hyun JW and Kim HS: Phloroglucinol exerts protective effects against oxidative stressinduced cell damage in SH-SY5Y cells. J Pharmacol Sci 119: 186-192, 2012.

20. Lin T, Liu Y, Shi M, Liu X, Li L, Liu Y and Zhao G: Promotive effect of ginsenoside Rd on proliferation of neural stem cells in vivo and in vitro. J Ethnopharmacol 142: 754-761, 2012. 